\title{
Shape of the carbon monoxide infrared absorption band of carboxyheme proteins as a probe of the protein anharmonicity
}

\author{
Solomon S. Stavrov \\ Sackler Institute of Molecular Medicine, Department of Human Molecular Genetics and Biochemistry, \\ Sackler Faculty of Medicine, Tel Aviv University, Ramat Aviv, Tel Aviv, 69978, Israel \\ Tel.: +972(3)640 9859; Fax: +972(3)640 5168; E-mail: stavrov@post.tau.ac.il
}

\begin{abstract}
Theory of the CO infrared absorption band of carbonmonoxyheme proteins is developed using results of the theory of optical absorption bandshape of impurity center in crystal. It is shown that the bandshape is controlled by electrostatic interaction to the polar or/and charged heme surrounding. Analysis of the $\mathrm{CO}$ bands of different heme proteins brings us to conclusion, that the CO band is broadened by very slow $(\tau>10 \mathrm{ps})$ motions of the heme surrounding and this motion most probably corresponds to the slow collective motion of the protein molecule. Therefore the second moment of the band must depend linearly on temperature at $T>25 \mathrm{~K}$ if the heme surrounding moves harmonically. The motion of the protein formed surrounding of the heme is arrested by the glassy protein environment. It is shown that Gaussian is the only possible symmetric shape of the CO band, if the heme surrounding moves harmonically. Deviation from this bandshape is a manifestation of anharmonic character of the surrounding motion. In general, CO infrared absorption band is shown to be an excellent probe of the dynamics of the heme surrounding.
\end{abstract}

Keywords: Protein dynamics, myoglobin, glycerol, heme proteins

\section{Introduction}

Dynamics of biological molecules are studied very intensively by virtually all methods of modern chemistry and physics. Special role in these studies play heme proteins (HPs), because their active center, heme, can be studied by virtually all spectroscopic techniques. In particular, it was kinetic measurements of $\mathrm{CO}$ recombination after photolysis of carbonmonoxymyoglobin $(\mathrm{Mb}(\mathrm{CO}))$ that lead to the view of conformational substates (CSS) and energy barriers [2,3,9,10]. Population of CSSs is very important for the protein function and leads to strongly anharmonic motion of proteins [14]. Therefore, study of the transformation of the protein motion from the harmonic to anharmonic and dependence of this transformation on the protein structure, temperature of the sample and its state (liquid or solid) is studied very intensively, see, for example, [7,10,30].

Infrared absorption (IR) spectra of carbon monoxide molecule coordinated by the heme iron of carbonmonoxyheme proteins, $\mathrm{HP}(\mathrm{CO})$, were intensively used to study structure and dynamics $[2,6,15-17$, $21,28,33,35,36]$ of different $\mathrm{HP}(\mathrm{CO}) \mathrm{s}$. This band corresponds to transition between the ground and first 
excited vibrational states of a normal vibration, which has the major contribution of the $\mathrm{C}-\mathrm{O}$ valence vibration of the coordinated $\mathrm{CO}$ molecule. The band is well isolated from other protein IR bands, its position varying from 1900 through $2000 \mathrm{~cm}^{-1}$ in different $\mathrm{HP}(\mathrm{CO}) \mathrm{s}$. In some of them the band consists of several components, each of which is usually assigned to different CSSs of the protein. Modern techniques provide one with reliable information not only on position of the $\mathrm{CO}$ band, but also on its shape, see, for example, $[2,16,17,28,36]$. The shift in the $\mathrm{CO}$ band position is caused by electrostatic interaction with the heme surrounding (HS), whereas the HS motion causes the band's broadening [13,16,18-21, 25].

Contribution of different interactions into shape of a vibrational band was studied in a number of publications $[1,4,5,11,12,22,24,29,34]$. First, resonant transfer of vibrational energy between identical molecules causes exciton-like broadening of the band. However, in the case of active centers buried in proteins, this interaction is negligibly weak. Second, there is natural broadening, which stems from decay of excited vibrational state. In carbonyl complexes of transition metals this broadening is less than $0.2 \mathrm{~cm}^{-1}$ even at room temperature [27] and, consequently, hardly contributes to the shape of the CO band of $\mathrm{HP}(\mathrm{CO})$, full width at half maximum $(\Gamma)$ of which is larger $[2,8,16,17,20]$ than $10 \mathrm{~cm}^{-1}$. Third, energy levels of a molecule fluctuate adiabatically due to changes in the interaction of the molecule with its surroundings; its resulting fluctuations manifest itself in the band broadening. This interaction, which sometimes is called pure dephasing, mainly contributes to the CO bandshape $[16,17,25-27,36]$.

In this paper we develop theory of the $\mathrm{CO}$ bandshape and its temperature dependence assuming that the heme surrounding moves harmonically. The results are used to interpret the experimental data and to reveal at which temperatures and in what protein environment the harmonic motion of the HS transforms into the anharmonic one. It is shown that the $\mathrm{CO}$ band is a reliable probe of this transition.

\section{Interaction of the $\mathrm{CO}$ vibration and the heme surrounding}

Previously we showed $[18,19,36]$ that broadening of the CO band is controlled by electrostatic interaction of moving charged or/and polar parts of HS. This interaction was shown to be relatively weak: $1 \AA$ shift of a unit point charge changes the CO frequency by the order of $10 \mathrm{~cm}^{-1}$. This implies that there must be weak coupling between the $\mathrm{C}-\mathrm{O}$ stretching coordinate and the HS motion.

If both the heme-CO and HS move harmonically, this coupling can be described by the Hamiltonian

$$
\begin{aligned}
& H=H_{0}+H_{\mathrm{int}}, \\
& H_{0}=-\frac{\hbar^{2}}{2 M} \frac{\partial^{2}}{\partial Q^{2}}-\sum_{i} \frac{\hbar^{2}}{2 m_{i}} \frac{\partial^{2}}{\partial q_{i}^{2}}+\frac{1}{2} M \Omega_{0}^{2} Q^{2}+\frac{1}{2} \sum_{i} m_{i} \omega_{i}^{2} q_{i}^{2}, \\
& H_{\text {int }}=M \Omega_{0}^{2} Q^{2} \sum_{i} \alpha_{i} q_{i},
\end{aligned}
$$

where $H_{0}$ is Hamiltonian of a number of non-interacting oscillators; $M$ and $m_{i}, \Omega$ and $\omega_{i}$, and $Q$ and $q_{i}$, are moments, reduced masses, frequencies, and displacements along the normal coordinates consisting mainly of the $\mathrm{C}-\mathrm{O}$ distance and the HS modes, respectively. $H_{\text {int }}$ describes the coupling between these subsystems, and $\alpha_{i}$ accounts for its strength. 
Since the CO subsystem is much faster than the HS subsystem, the adiabatic approximation [23,31,32] can be used to find the eigenvalues and eigenfunctions of $H$ (1)

$$
\begin{aligned}
& E_{N,\left\{n_{i}\right\}}=\hbar \Omega_{0}\left(N+\frac{1}{2}\right)+\sum_{i} \hbar \omega_{i}\left(n_{i}+\frac{1}{2}\right)-\frac{1}{2} \hbar \Omega_{0} \xi\left(N+\frac{1}{2}\right)^{2}, \\
& \Psi_{N,\left\{n_{i}\right\}}(Q, q)=\Phi_{N}\left(Q, q_{1}, \ldots, q_{i}, \ldots, q_{j}\right) \prod_{n_{i}} \varphi_{n_{i}}\left(q_{i}-q_{i N}\right),
\end{aligned}
$$

where

$$
q_{i N}=-\frac{\xi_{i}}{\alpha_{i}}\left(N+\frac{1}{2}\right), \quad \xi_{i}=\frac{\hbar \Omega_{0} \alpha_{i}^{2}}{m_{i} \omega_{i}^{2}}, \quad \xi=\sum_{i} \xi_{i}
$$

and $N$ and $n_{i}$ are vibrational quantum numbers of the CO and HS subsystems, respectively.

\section{Shape of the CO band}

Even at relatively high temperatures excited states of the CO subsystem are hardly populated, because the energy gap between the ground and first excited state of this subsystem is about $2000 \mathrm{~cm}^{-1}$. Therefore, only $0, n \rightarrow 1, n^{\prime}$ transitions notably contribute to the $\mathrm{CO}$ band.

Probabilities of the $0, n \rightarrow 1, n^{\prime}$ transitions are calculated in the dipole approximation. Using Condon approximation [23,32], neglecting dispersion of the frequencies of the HS subsystem and summing over all the $0, n \rightarrow 1, n^{\prime}$ transitions one obtains [23,31,32]

$$
F_{0 \rightarrow 1}(\Omega) \sim \exp \left[-S \operatorname{coth}\left(\frac{\beta_{0}}{2}\right)\right] \sum_{p} \exp \left(p \frac{\beta_{0}}{2}\right) I_{p}\left[\frac{S}{\sinh \left(\beta_{0} / 2\right)}\right] \delta\left[\Omega-\Omega_{0}(1-\xi)-p \omega_{0}\right],
$$

where $I_{p}$ is the modified Bessel function of the first kind, $S$ is Huang-Rhys constant

$$
S=\frac{1}{2} \frac{\Omega_{0}}{\omega_{0}} \xi, \quad \beta_{0}=\frac{\hbar \omega_{0}}{k_{\mathrm{B}} T},
$$

$k_{\mathrm{B}}$ is Boltzmann constant, and $T$ is the sample temperature.

The natural broadening of the excited vibrational state of the CO subsystem can be taken into account [31,32] by substituting $\delta$ function in (4) for Lorentzians centered at $\Omega_{0}(1-\xi)-p \omega_{0}$.

It is convenient to describe shape of the band using method of moments [23,32]

$$
\begin{aligned}
& M_{0}=\int_{-\infty}^{\infty} F(\Omega) \mathrm{d} \Omega, \quad M_{1}=\frac{1}{M_{0}} \int_{-\infty}^{\infty} F(\Omega) \Omega \mathrm{d} \Omega, \\
& M_{n(n>1)}=\frac{1}{M_{0}} \int_{-\infty}^{\infty} F(\Omega)\left(\Omega-M_{1}\right)^{n} \mathrm{~d} \Omega,
\end{aligned}
$$

which can be calculated analytically even in the case of strong dispersion. It was shown [23,32], that in the case under consideration $M_{0}$ and $M_{1}$ (which are intensity of the band and its center of gravity, 
respectively) are temperature independent, whereas $M_{2}$ (which is proportional to $\Gamma^{2}$ ) has very specific temperature dependence

$$
M_{2}=M_{20}+\frac{1}{2} \Omega_{0} \sum_{i} \xi_{i} \omega_{i} \operatorname{coth}\left(\frac{\beta_{i}}{2}\right)
$$

where $M_{20}$ accounts for the temperature independent inhomogeneous broadening of the band [23,32].

\section{Discussion}

It follows from the previous section, that broadening of the band is caused by presence of a number of the $n_{i} \rightarrow n_{i}^{\prime}$ transitions; the more such transitions essentially contribute to the band, the wider band is. Hence, $\omega_{i}<\Gamma$. In $\mathrm{Mb}(\mathrm{CO})$ even at room temperature $\Gamma \approx 10 \mathrm{~cm}^{-1}$ [2]; consequently, $\omega_{i}<10 \mathrm{~cm}^{-1}$ and characteristic time of the HS motion $\tau>3$ ps. Since the band is formed by more than two $n \rightarrow n^{\prime}$ transitions, one can reliably state that $\tau>10 \mathrm{ps}$.

This result, first of all, strongly justifies use of the adiabatic approximation to find eigenvectors and eigenfunctions of Hamiltonian (1), because $\Omega_{0}\left(\sim 2000 \mathrm{~cm}^{-1}\right)$ is much larger than $\omega_{i}\left(<10 \mathrm{~cm}^{-1}\right)$.

Second, $\tau$ is much longer, than characteristic times of internal vibrations of amino acids. Consequently these vibrations do not contribute to the broadening. Thus, the $\mathrm{CO}$ band is broadened by electrostatic interaction of the CO oscillator with some slowly moving parts of HS. If the heme pocket is not populated by solvent molecules (which can move slowly in the pocket), most probably these are motions of big parts of the protein, these parts participate in slow collective motion (SCM) of the protein molecule.

A plausible candidate for such a motion can be shift of the $\mathrm{E}$ helix in respect to the $\mathrm{F}$ helix. Indeed, in $\mathrm{Mb}(\mathrm{CO})$ histidine 64 (distal histidine) is polar and is a part of the E helix, whereas heme is covalently bonded to the $\mathrm{F}$ helix through histidine 93 . Motion of the $\mathrm{E}$ helix with respect to the $\mathrm{F}$ helix would change the distance between the distal histidine and the heme, changing the histidine electric field on the heme-CO complex, affecting its electronic structure, and, as a result, broadening the $\mathrm{CO}$ band.

This picture corroborate with our previous conclusion $[18,19,36]$ that the CO band can be broadened only by motions, which include large (in respect to the heme) displacements of charged or polar residues. It is clear, that these motions are expected to be much slower than internal vibrations of amino acids.

Third, because of such small values of $\omega_{i}$, criterion $\beta_{i} / 2 \ll 1$ fulfils at $T>25 \mathrm{~K}$ and for these temperatures expression (7) can be rewritten

$$
M_{2}=M_{20}+\Omega_{0} \xi k_{\mathrm{B}} T .
$$

However, experimental studies of myoglobin [2], $\mathrm{Mb}(\mathrm{CO})$, and hemoglobin [8], $\mathrm{Hb}(\mathrm{CO})$, at $\mathrm{pH} \sim 7$ in glycerol/water solvents undergoing glass-liquid transition at $T_{c} \approx 160-200 \mathrm{~K}$ showed that $M_{2}$ linearly depends on $T$ only in the liquid environment at $T>T_{c}$ and is constant at $T<T_{c}$, Fig. 1.

To understand cause of this deviation from the linearity we have to analyze assumptions made in the second and third sections: (a) describing interaction between the CO and HS subsystems we included in $H_{\text {int }}$ (1) linear in $q$ terms and neglected the higher order terms; (b) we used Condon approximation; and (c) we assumed that HS moves harmonically.

If any one of the first two assumptions was wrong, one would observe non-linear dependence of $M_{2}$ on temperature: the higher is the temperature, the stronger is non-linearity $[23,31,32]$. The experimental 


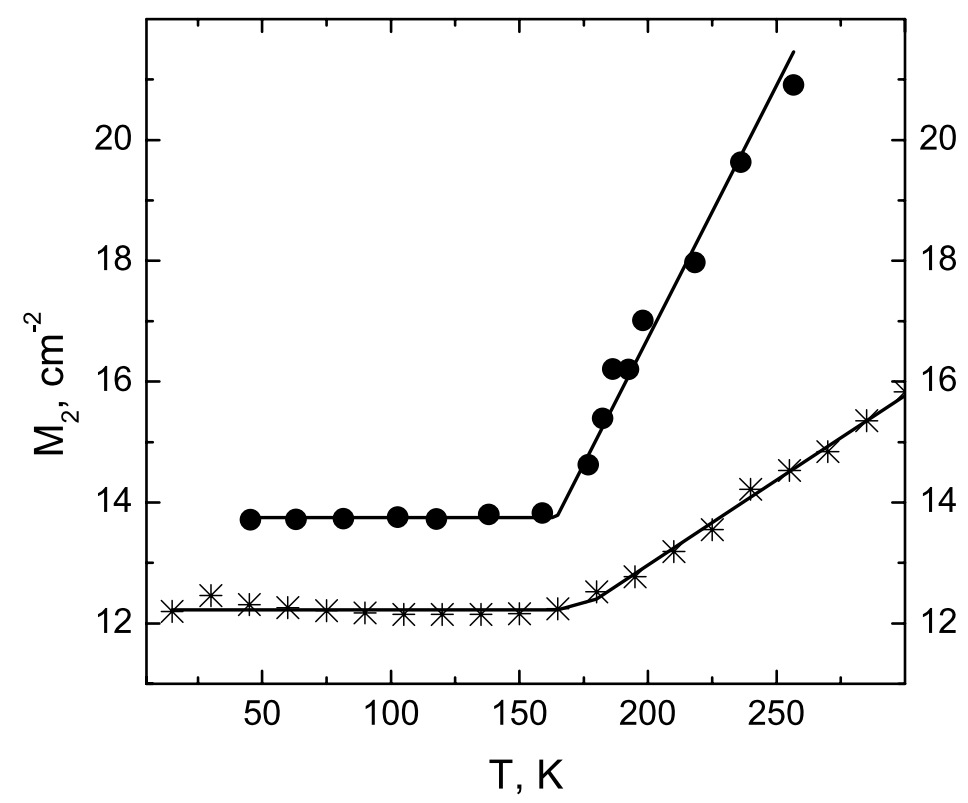

Fig. 1. Experimentally observed temperature dependences of the $M_{2}$ of different $\mathrm{HP}(\mathrm{CO}) \mathrm{s}$ (symbols) in glycerol/water solvents and their fits (lines) using (9): $\mathrm{Mb}(\mathrm{CO})$ [2] at $\mathrm{pH} 6.8$ (circles, $M_{20}=0, \Omega_{0} \xi k_{\mathrm{B}}=0.12 \mathrm{~cm}^{-1}, T_{c}=165 \mathrm{~K}$ ) and $\mathrm{Hb}(\mathrm{CO})$ [8] at pH 6.8 (asterisks, $M_{20}=0, \Omega_{0} \xi k_{\mathrm{B}}=0.12 \mathrm{~cm}^{-1}, T_{c}=165 \mathrm{~K}$ ).

data show the totally different temperature dependence: $M_{2}$ depends linearly on $T$ at temperatures $T>$ $T_{c}$ (strongly supporting use of these approximations), whereas at lower temperatures it is constant.

Consequently, we conclude that the assumption (c) of harmonic motion of HS in the whole interval of temperatures was wrong: at $T>T_{c}$ HS moves harmonically, whereas at $T<T_{c}$ the motions, which broaden the $\mathrm{CO}$ band, are frozen. This can happen if the protein motions contributing into the $\mathrm{CO}$ broadening are arrested by the glassy environment. This explanation seems to be reasonable, because it is natural to assume, that SCMs of the protein globule (in particular, relative motions of two helixes) have to be sensitive to the protein environment.

Taking into account (8), this conclusion can be formulated mathematically as follows

$$
\begin{aligned}
& M_{2}=M_{20}+\Omega_{0} \xi k_{\mathrm{B}} T_{\text {eff }}, \\
& T_{\text {eff }}=T_{c}, \quad T<T_{c}, \\
& T_{\text {eff }}=T, \quad T \geqslant T_{c},
\end{aligned}
$$

this expression allows to interpret the experimental data quantitatively, see Fig. 1.

Analysis of bandshape (4) showed [23,31,32] that upon heating the shape of the band becomes more symmetric and if the temperature is high enough the bandshape becomes Gaussian.

In principle, effect of natural broadening of each component of the band could transform the bandshape into Voigtian, which is a convolution of Gaussian and Lorentzian. However, the natural width [27] of each line is less than $0.2 \mathrm{~cm}^{-1}$, which is much less, than total width of the band $\Gamma \geqslant 10 \mathrm{~cm}^{-1}$. Consequently, the $\mathrm{CO}$ band can have the only possible symmetric shape - Gaussian. Considerable deviation of a symmetric CO bandshape from Gaussian (see, for example, [28]) can be a manifestation of thermal population of higher-energy CSSs, which transforms the protein motion in the anharmonic one. 


\section{Conclusion}

We presented above theory of shape of the isolated infrared absorption band, which corresponds to the absorption of a higher-frequency oscillator linearly coupled to a number of low-frequency oscillators. We showed that this problem is analogical to the problem of optical absorption spectrum of impurity center.

The results allowed to make a number of conclusions for the case of $\mathrm{HP}(\mathrm{CO}) \mathrm{s}$ :

(a) The CO infrared absorption band is broadened by very slow ( $\tau>10 \mathrm{ps)} \mathrm{motions} \mathrm{of} \mathrm{HS.}$

(b) In the case of harmonic protein motion $M_{2}$ of this band must depend on temperature linearly at $T>25 \mathrm{~K}$.

(c) This HS motion, most probably, is a component of protein SCMs.

(d) Transition liquid $\rightarrow$ glass arrests SCMs, this arrest is manifested in the independence of $M_{2}$ on temperature at $T<T_{c}$.

(e) Gaussian is the only possible symmetric shape of the CO band. Therefore observation of nonGaussian IR absorption band (Voigtian, for example) hints at the population of a number of the protein CSSs and anharmonic character of the protein motion. To clarify the situation the temperature dependence experiments are necessary.

The results obtained in this paper are applicable to the complexes with other ligands like $\mathrm{NO}$ and $\mathrm{O}_{2}$. Simplicity of the theoretical interpretation of this band makes it an excellent probe of the HS dynamics.

\section{Acknowledgements}

The author is grateful to Dr. G. Uli Nienhaus for discussions, which stimulated this study and Dr. Boris S. Tsukerblat for discussions of the results.

\section{References}

[1] R.J. Abbott and D.W. Oxtoby, Exchange dephasing and motional narrowing of vibrational-modes, J. Chem. Phys. 70 (1979), 4703-4707.

[2] A. Ansari, J. Berendzen, D. Braunstein, B.R. Cowen, H. Frauenfelder, M.K. Hong, I.E.T. Iben, J.B. Johnson, P. Ormos, T.B. Sauke, R. Scholl, A. Schulte, P.J. Steinbach, J. Vittitow and R.D. Young, Rebinding and relaxation in the myoglobin pocket, Biophys. Chem. 26 (1987), 337-355.

[3] R.H. Austin, K.W. Beeson, L. Eisenstein, H. Frauenfelder and I.C. Gunsalus, Dynamics of ligand binding to myoglobin, Biochemistry 14 (1975), 5355-5373.

[4] S. Bratos and E. Marechal, Raman study of liquids. I. Theory of the Raman spectra of diatomic molecules in inert solutions, Phys. Rev. A 4 (1971), 1078-1092.

[5] S. Bratoz, J. Rios and Y. Guissani, Infrared study of liquids. I. The theory of the IR spectra of diatomic molecules in inert solutions, J. Chem. Phys. 52 (1970), 439-453.

[6] L. Cordone, G. Cottone, S. Giuffrida and F. Librizzi, Thermal evolution of the CO stretching band in carboxy-myoglobin in the light of neutron scattering and molecular dynamics simulations, Chem. Phys. 345 (2008), 275-282.

[7] L. Cordone, G. Cottone, S. Giuffrida, G. Palazzo, G. Venturoli and C. Viappiani, Internal dynamics and protein-matrix coupling in trehalose-coated proteins, BBA - Proteins Proteomics 1749 (2005), 252-281.

[8] A. Cupane, M. Leone and V. Militello, Conformational substates and dynamic properties of carbonmonoxy hemoglobin, Biophys. Chem. 104 (2003), 335-344.

[9] A. Cupane, M. Leone, E. Vitrano and L. Cordone, Structural and dynamic properties of the heme pocket in myoglobin probed by optical spectroscopy, Biopolymers 27 (1988), 1977-1997.

[10] D. Dantsker, U. Samuni, J.M. Friedman and N. Agmon, A hierarchy of functionally important relaxations within myoglobin based on solvent effects, mutations and kinetic model, BBA - Proteins Proteomics 1749 (2005), 234-251. 
[11] D.J. Diestler, Vibrational phase and energy relaxation in broadening of Raman spectral lines in dense media, Chem. Phys. Lett. 39 (1976), 39-44.

[12] M.D. Fayer, Fast protein dynamics probed with infrared vibrational echo experiments, Annu. Rev. Phys. Chem. 52 (2001), 315-356.

[13] S. Franzen, An electrostatic model for the frequency shifts in the carbonmonoxy stretching band of myoglobin: Correlation of hydrogen bonding and the Stark tuning rate, J. Amer. Chem. Soc. 124 (2002), 13271-13281.

[14] H. Frauenfelder, P.W. Fenimore and R.D. Young, Protein dynamics and function: Insights from the energy landscape and solvent slaving, IUBMB Life 59 (2007), 506-512.

[15] H. Frauenfelder, G.U. Nienhaus and R.D. Young, Relaxation and disorders in proteins, in: Disorder Effects on Relaxation Processes, R. Richert and A. Blumen, eds, Springer-Verlag, Berlin, 1994, pp. 592-614.

[16] A.D. Kaposi, N.V. Prabhu, S.D. Dalosto, K.A. Sharp, W.W. Wright, S.S. Stavrov and J.M. Vanderkooi, Solvent dependent and independent motions of CO-horseradish peroxidase examined by infrared spectroscopy and molecular dynamics calculations, Biophys. Chem. 106 (2003), 1-14.

[17] A.D. Kaposi, J.M. Vanderkooi and S.S. Stavrov, Infrared absorption study of the heme pocket dynamics of carbonmonoxyheme proteins, Biophys. J. 91 (2006), 4191-4200.

[18] B. Kushkuley and S.S. Stavrov, Theoretical study of the distal-side steric and electrostatic effects on the vibrational characteristics of the FeCO unit of the carbonylheme proteins and their models, Biophys. J. 70 (1996), 1214-1229.

[19] B. Kushkuley and S.S. Stavrov, Theoretical study of the electrostatic and steric effects on the spectroscopic characteristics of the metal-ligand unit of heme proteins. 2. C-O vibrational frequencies, O-17 isotropic chemical shifts, and nuclear quadrupole coupling constants, Biophys. J. 72 (1997), 899-912.

[20] T. Li, M.L. Quillin, G.N. Phillips and J.S. Olson, Structural determinants of the stretching frequency of CO bound to myoglobin, Biochemistry 33 (1994), 1433-1446.

[21] X.Y. Li and T.G. Spiro, Is bound CO linear or bent in heme-proteins - evidence from resonance Raman and infrared spectroscopic data, J. Amer. Chem. Soc. 110 (1988), 6024-6033.

[22] P.A. Madden and R.M. Lyndenbell, Theory of vibrational linewidths, Chem. Phys. Lett. 38 (1976), 163-165.

[23] J.J. Markham, Interaction of normal modes with electron traps, Reviews of Modern Physics 31 (1959), 956-989.

[24] S. Marks, P.A. Cornelius and C.B. Harris, A critical test of vibrational dephasing theories in solids using spontaneous Raman scattering in isotropically mixed crystals, J. Chem. Phys. 73 (1980), 3069-3081.

[25] K.A. Merchant, W.G. Noid, R. Akiyama, I.J. Finkelstein, A. Goun, B.L. McClain, R.F. Loring and M.D. Fayer, Myoglobin-CO substate structures and dynamics: Multidimensional vibrational echoes and molecular dynamics simulations, J. Amer. Chem. Soc. 125 (2003), 13804-13818.

[26] K.A. Merchant, W.G. Noid, D.E. Thompson, R. Akiyama, R.F. Loring and M.D. Fayer, Structural assignments and dynamics of the A substates of MbCO: Spectrally resolved vibrational echo experiments and molecular dynamics simulations, J. Phys. Chem. B 107 (2003), 4-7.

[27] K.A. Merchant, D.E. Thompson, Q.H. Xu, R.B. Williams, R.F. Loring and M.D. Fayer, Myoglobin-CO conformational substate dynamics: 2D vibrational echoes and MD simulations, Biophys. J. 82 (2002), 3277-3288.

[28] J.D. Muller, B.H. McMahon, E.Y.T. Chien, S.G. Sligar and G.U. Nienhaus, Connection between the taxonomic substates and protonation of histidines 64 and 97 in carbonmonoxy myoglobin, Biophys. J. 77 (1999), 1036-1051.

[29] D.W. Oxtoby and S.A. Rice, Vibrational relaxation in liquids, Chem. Phys. Lett. 42 (1976), 1-7.

[30] F.G. Parak, Physical aspects of protein dynamics, Reports on Progress in Physics 66 (2003), 103-129.

[31] Y.E. Perlin, Modern methods in the theory of many-phonon processes, Sov. Phys. Uspekhi 80 (1964), 553-595.

[32] Y.E. Perlin and B.S. Tsukerblat, Effects of Electron-Vibrational Interactions in Optical Spectra of Paramagnetic Impurities, Shtinnitsa, Kishenev, 1974.

[33] W.T. Potter, J.H. Hazzard, M.G. Choc, M.P. Tucker and W.S. Caughey, Infrared spectra of carbonyl hemoglobins: Characterization of dynamic heme pocket conformers, Biochemistry 29 (1990), 6283-6295.

[34] R.M. Shelby, C.B. Harris and P.A. Cornelius, The origin of vibrational dephasing of polyatomic molecules in condensed phases, J. Chem. Phys. 70 (1979), 34-41.

[35] T.G. Spiro and I.H. Wasbotten, CO as a vibrational probe of heme protein active sites, J. Inorg. Biochem. 99 (2005), 34-44.

[36] S.S. Stavrov, The FeCO unit vibrations as a probe of the structure and dynamics of the active site of heme proteins: combined quantum chemical, vibronic and spectroscopic study, in: Biopolymer Research Trends, T.S. Németh, ed., Nova Publishers, New York, 2008, pp. 119-143. 


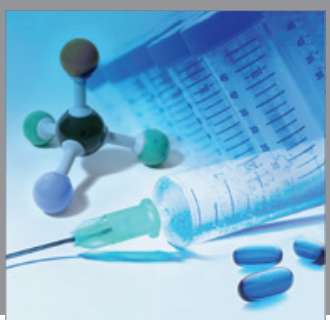

International Journal of

Medicinal Chemistry

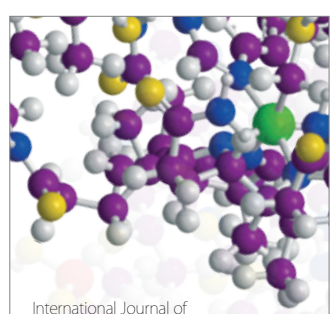

Carbohydrate Chemistry

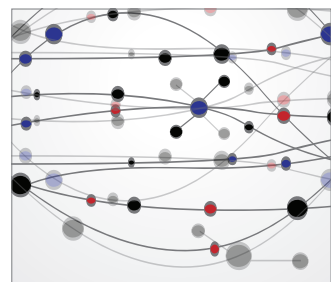

The Scientific World Journal
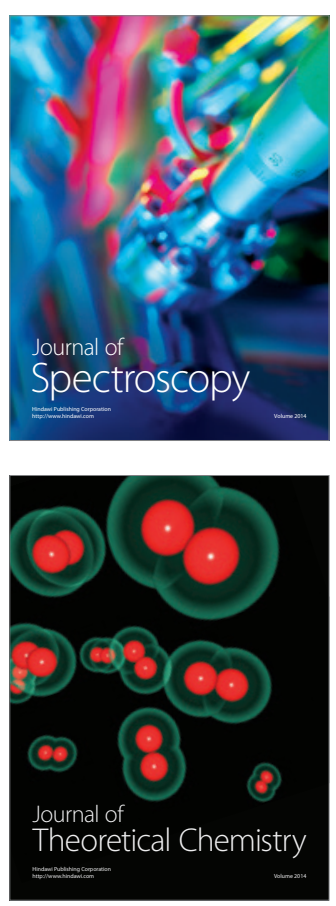
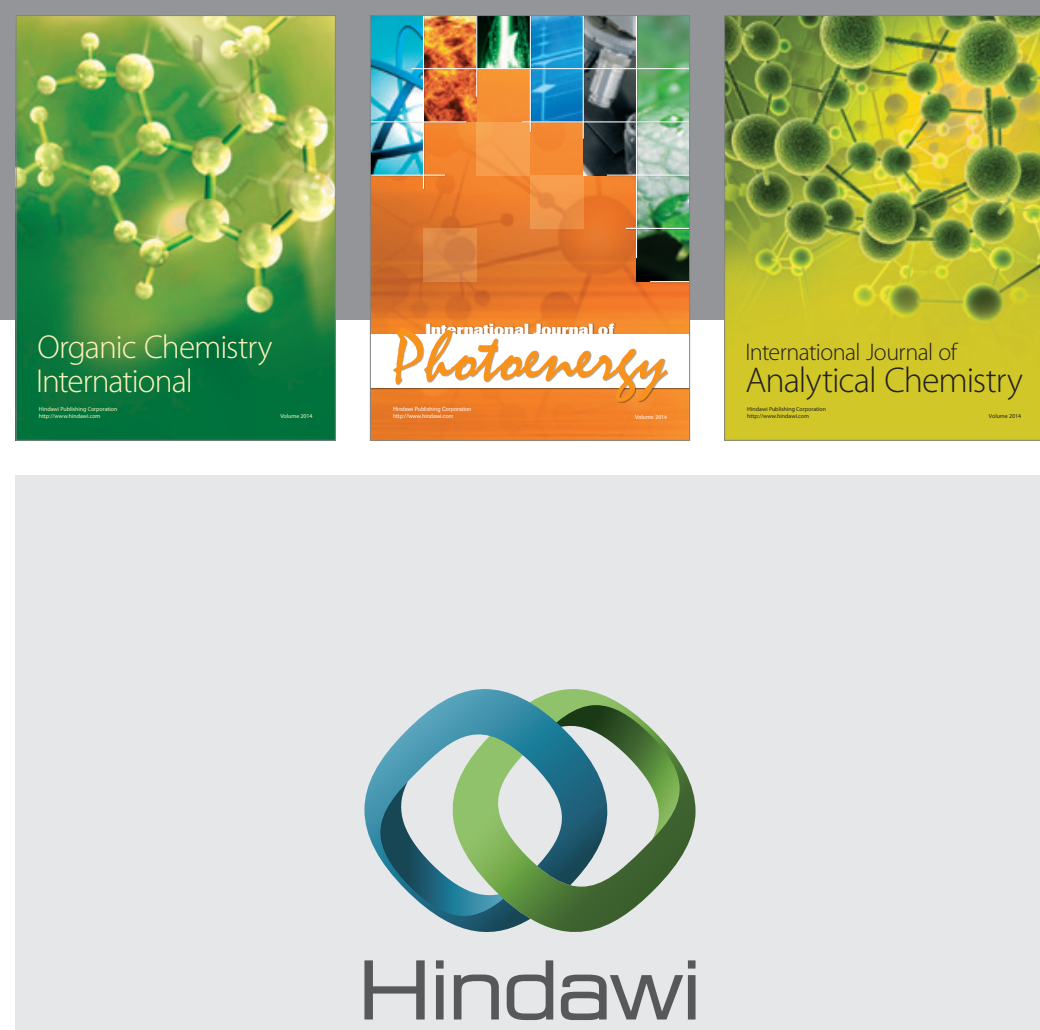

Submit your manuscripts at

http://www.hindawi.com
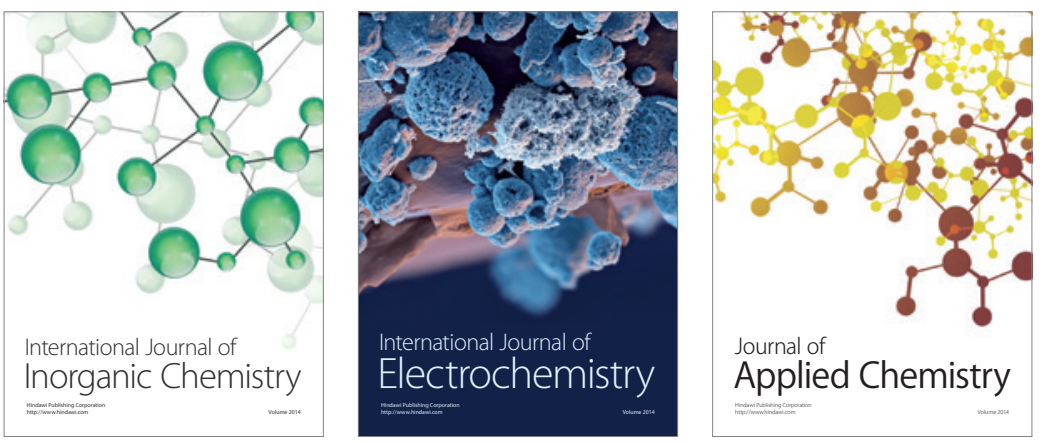

Journal of

Applied Chemistry
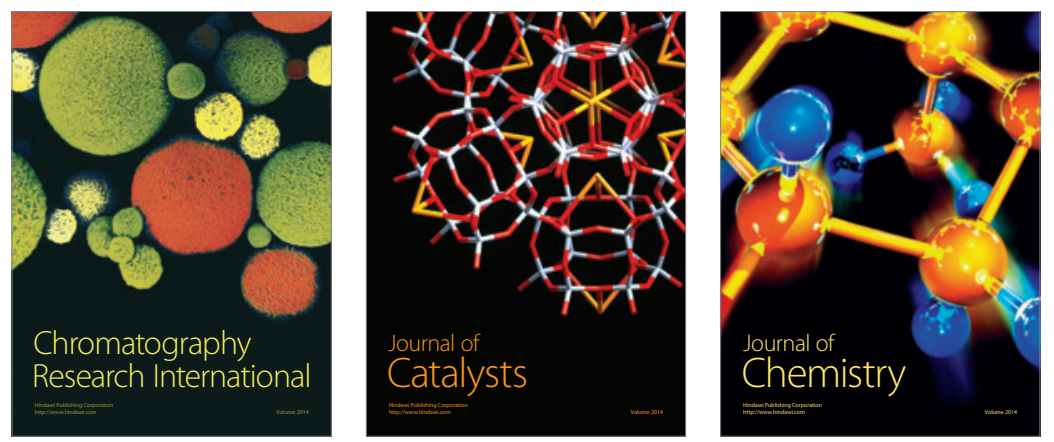
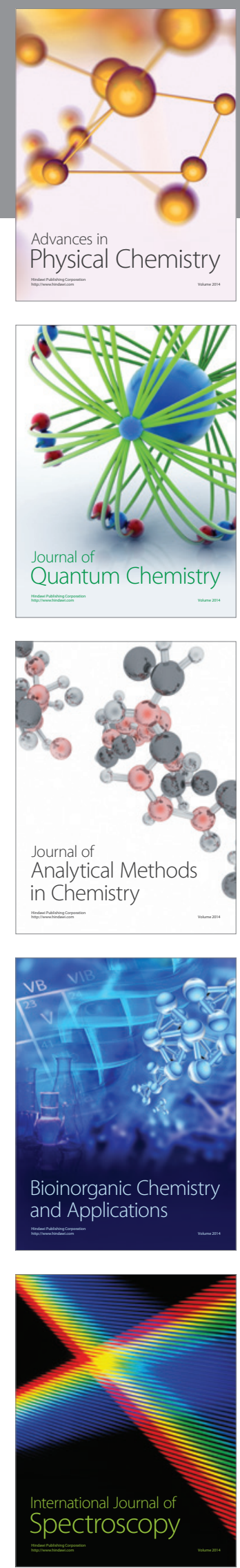